\title{
Improvement of stilbene production by mulberry Morus alba root culture via precursor feeding and co-elicitation
}

\author{
Chadathorn Inyai ${ }^{1,2} \cdot$ Gorawit Yusakul $^{3}$ (1) $\cdot$ Jukrapun Komaikul $^{4} \cdot$ Tharita Kitisripanya $^{5}$ (D) Kittisak Likhitwitayawuid $^{6}$. \\ Boonchoo Sritularak ${ }^{6}\left(\mathbb{D} \cdot\right.$ Waraporn Putalun ${ }^{1,2}$ (1)
}

Received: 11 July 2020 / Accepted: 26 October 2020 / Published online: 10 November 2020

(c) Springer-Verlag GmbH Germany, part of Springer Nature 2020

\begin{abstract}
Large amounts of Morus alba L. (MA) roots are needed as the source of active stilbenes in the industrial production of traditional medicines and cosmeceuticals. A recent investigation demonstrated resveratrol and its derivatives to be promising anti-COVID-19 agents. However, conventional cultivation of MA does not meet the demand for its stilbenes, and root quality usually varies between crops. This study established the in vitro non-GMO root culture of MA and optimized the root density, precursor feeding, and elicitors for stilbene productivity. A root culture with optimal inoculum density ( $3 \mathrm{~g} /$ flask of $30 \mathrm{~mL}$ medium) accumulated mulberroside A, oxyresveratrol, and resveratrol at $18.7 \pm 1.00 \mathrm{mg} / \mathrm{g}, 136 \pm 5.05 \mu \mathrm{g} / \mathrm{g}$, and $41.6 \pm 5.84 \mu \mathrm{g} / \mathrm{g}$ dry weight (DW), respectively. The feeding of L-tyrosine shortened the time required to reach the stilbene productive stage. Root cultures co-treated with $200 \mu \mathrm{M}$ methyl jasmonate and $2 \mathrm{mg} / \mathrm{mL}$ yeast extract accumulated the highest contents of mulberroside A $(30.3 \pm 2.68 \mathrm{mg} / \mathrm{g} \mathrm{DW})$, oxyresveratrol $(68.6 \pm 3.53 \mu \mathrm{g} / \mathrm{g} \mathrm{DW})$, and resveratrol $(10.2 \pm 0.53 \mu \mathrm{g} / \mathrm{g}$ DW). In summary, root culture is a promising and sustainable source of stilbenes for the development of health products and agents for further investigation as potential anti-COVID-19 agents.
\end{abstract}

Keywords Morus alba $\cdot$ Root culture $\cdot$ Stilbene $\cdot$ Elicitation $\cdot$ Resveratrol

Electronic supplementary material The online version of this article (https://doi.org/10.1007/s00449-020-02474-7) contains supplementary material, which is available to authorized users.

\section{Waraporn Putalun}

waraporn@kku.ac.th

1 Faculty of Pharmaceutical Sciences, Khon Kaen University, Khon Kaen 40002, Thailand

2 Research Group for Pharmaceutical Activities of Natural Products Using Pharmaceutical Biotechnology (PANPB), National Research University-Khon Kaen, Khon Kaen, Thailand

3 School of Pharmacy, Walailak University, Nakhon Si Thammarat, Thaiburi, Thasala, Thailand

4 Institute of Molecular Biosciences, Mahidol University, Bangkok, Thailand

5 Faculty of Pharmacy, Mahidol University, Bangkok, Thailand

6 Faculty of Pharmaceutical Sciences, Chulalongkorn University, Bangkok, Thailand

\section{Introduction}

In Thailand, Morus alba L. (MA) leaves are an essential food source for silkworm larvae due to their high protein and carbohydrate contents. MA roots contain high levels of bioactive stilbene compounds, including mulberroside A (MuA), oxyresveratrol (OXY), and resveratrol (RES), and thus are an important source of stilbenes for the health product industry. MA roots have also been utilized commercially as an active ingredient in whitening and anti-aging cosmetics. Studies have reported these stilbene compounds to have anti-inflammatory [1], anti-tyrosinase [2], and antioxidant $[3,4]$ properties. Moreover, MA root bark has been used as an ingredient in Chinese Herbal Pharmacopoeia recipes [5]. Because MA phytochemicals exhibit various pharmacological activities related to the oxidative process and aging, MA is in high demand in the industrial production of cosmetic and health-promoting products. During the COVID-19 pandemic, this natural product has also received attention in the search for new drugs, as stilbenes provide pharmacological activity against COVID-19 pathology. Via molecular dynamics simulation, RES has been shown to interact with 
viral spike protein [6], and the synthetic isomer of OXY has shown an inhibitory effect on SARS coronavirus replication [7]. Moreover, RES and OXY are reactive against other pathogenic viruses $[8,9]$. Thus, stilbenes are useful compounds against the SARS coronavirus.

In general, the cultivation of the mulberry tree requires a lengthy period before the large root can be collected. In addition, the season of harvest affects the production level of secondary metabolites [4]. Environmental factors (temperature, soil nutrients, rainfall, etc.) usually influence secondary metabolism and bioactive accumulation in medicinal plants. Therefore, an alternative cultivation system is required to solve the limitations of the MA source and its quality variation. A system of in vitro plant tissue culture is one method of choice, in which the plant is cultured in a synthetic medium under controlled conditions. Such a technique could provide rapid propagation and high accumulation of the desired secondary metabolites. MA culturing systems, including callus, cell suspension, and root cultures, have been reported [10-12]. The treatment of callus cultures using 2-hydroxypropyl-beta-cyclodextrin has been shown to improve OXY and RES production [11]. Elicitation of MA cell suspension cultures with methyl jasmonate (MJ) and yeast extract (YS) has also been shown to enhance OXY and RES production [10].

Elicitation has proven to be effective for increasing stilbene production from MA in an in vitro culture. Elicitation targets the biosynthetic pathway(s) through which enzyme expression is altered. Each elicitor interacts with its receptor and influences the biosynthetic pathway in a specific manner [13]. A simultaneous treatment of elicitors therefore causes a synergized enhancement of secondary metabolite production [14].

Effective strategies for secondary metabolite production and accumulation involve not only elicitation but also the feeding of biosynthetic precursors $[15,16]$. Phenylalanine (PHE) and tyrosine (TYR) are precursors of stilbene production from the phenylpropanoid pathway [17]. To date, the effects of PHE and TYR feeding on stilbene production from MA root cultures have not been investigated. Furthermore, although YS, MJ, chitosan, and salicylic acid have been proven as effective elicitors in MA root cultures, their combination to enhance stilbene production has not yet been studied. Therefore, this study aimed to investigate the effects of precursor feeding and combined elicitation on the accumulation of stilbene compounds in MA root cultures. If effective, this treatment could be applied to large-scale MA root cultures to provide raw materials for important industries.

\section{Materials and methods}

\section{Chemicals}

Mulberroside A (MuA, a glycoside form of OXY) was purchased from Chengdu Biopurify, Ltd. (Sichuan, China), and oxyresveratrol (OXY, trans-2,4,3',5'-tetrahydroxystilbene) was purified by Assoc. Prof. Dr. Boonchoo Sritularak (Faculty of Pharmaceutical Sciences, Chulalongkorn University, Thailand). Other chemicals, including 2,2'-azino-bis (3-ethylbenzothiazoline-6-sulphonic acid) (ABTS), methyl jasmonate (MJ), yeast extract (YS), L-phenylalanine (PHE), L-tyrosine (TYR), bovine serum albumin (BSA), and resveratrol (RES, 3,4',5-trihydroxy-trans-stilbene), were purchased from Sigma-Aldrich Co. (MO, USA). The polyclonal antibody against MuA (MuA-pAb) was produced in a previous study [18]. Peroxidase (POD) anti-rabbit IgG antibody was purchased from MP Biomedical (CA, USA). All other chemical substances used in the experiment were of analytical grade.

\section{Establishment and treatment of root cultures}

\section{Plant materials}

The MA root cultures were obtained by cutting the roots of in vitro MA plantlets. The roots were cultured and maintained in a Murashige and Skoog (MS) liquid medium supplemented with $1 \mathrm{mg} / \mathrm{L} \alpha$-naphthaleneacetic acid (NAA), known as MS-NAA medium [12]. The cultures were shaken at $80 \mathrm{rpm}$ under a 16 -h photoperiod at $25 \pm 2{ }^{\circ} \mathrm{C}$.

\section{Root culture growth rate and stilbene accumulation}

The growth and stilbene accumulation of the MA root culture were evaluated over time. The 28-day-old roots were subcultured in $125-\mathrm{mL}$ flasks with $30 \mathrm{~mL}$ MS-NAA ( $1 \mathrm{~g} /$ flask). The cultures were shaken at $80 \mathrm{rpm}$ under a $16-\mathrm{h}$ photoperiod at $25 \pm 2{ }^{\circ} \mathrm{C}$. The cultured roots were harvested weekly, and their DWs were measured. The samples were dried in a hot air $\left(50{ }^{\circ} \mathrm{C}\right)$ oven over two days. Finally, the stilbene content was determined.

\section{Effect of root density on growth and stilbene accumulation}

The effect of root density on growth and stilbene accumulation was studied. In the experiment, roots with fresh weights of $0.5,1,2,3$, and $6( \pm 1 \%) \mathrm{g}$ were transferred into fresh 
MS-NAA (30 mL). After cultivation for 4 weeks, the roots and culture medium were harvested. The root samples were then dried and subjected to stilbene extraction for quantitative analysis of the stilbene content. The MuA content of the samples was determined by indirect competitive ELISA (icELISA), and the OXY and RES contents were determined by the HPLC-UV method. Each sample group was evaluated in triplicate. The growth index (GI) was calculated from the final fresh mass at the collection time (FFW) and initial fresh weight (IFW) of the cell suspension, as shown in the following equation.

$\mathrm{GI}=\frac{\mathrm{FFW}-\mathrm{IFW}}{\mathrm{FFW}}$

\section{Effect of precursor feeding on growth and stilbene accumulation}

A stock solution of PHE was prepared by dissolving PHE $(1.65 \mathrm{mg})$ in distilled water $(10 \mathrm{~mL})$, followed by filter sterilization. TYR $(1.81 \mathrm{mg})$ was dissolved in a few drops of $1.0 \mathrm{~N} \mathrm{HCl}$ and then diluted with distilled water to obtain $10 \mathrm{~mL}$ of $1 \mathrm{mM}$ stock solution, which was then filter-sterilized. The precursor concentrations were selected based on the concentrations that improved the accumulation of MuA, OXY, and RES in immobilized MA cell cultures in our previous experiment [10]. The precursors were added to the MA root culture at a final concentration of $0.05 \mathrm{mM}$ PHE, $0.03 \mathrm{mM}$ TYR, and a mixture of $0.05 \mathrm{mM}$ PHE and $0.03 \mathrm{mM}$ TYR (PT) on the first day of cultivation. The root samples were harvested in triplicate after 1, 5, 10, 20, 40, and 50 days of cultivation. The stilbene content of all samples was determined by icELISA and HPLC, as previously described.

\section{Elicitation of stilbene accumulation}

A stock solution of MJ (10 mM) was prepared in $40 \%(\mathrm{v} / \mathrm{v})$ aqueous ethanol and then filter-sterilized. For the YS stock solution, YS powder was dissolved in deionized water to make a $100 \mathrm{mg} / \mathrm{mL}$ stock solution. The $\mathrm{pH}$ of the stock solution was adjusted to 5.5, and the solution was then sterilized by autoclaving at $121^{\circ} \mathrm{C}$ and 15 psi for $15 \mathrm{~min}$. The MA root cultures were treated with elicitors on the 28th day of cultivation: YS $(2 \mathrm{mg} / \mathrm{mL}), \mathrm{MJ}(200 \mu \mathrm{M})$, a mixture of YS $(0.5 \mathrm{mg} / \mathrm{mL})$ and $\mathrm{MJ}(50 \mu \mathrm{M})$, a mixture of $\mathrm{YS}(1 \mathrm{mg} / \mathrm{mL})$ and MJ $(100 \mu \mathrm{M})$, or a mixture of YS $(2 \mathrm{mg} / \mathrm{mL})$ and MJ $(200 \mu \mathrm{M})$. The elicitor concentrations were selected according to the results of our previous experiments [12], in which a single treatment of $2 \mathrm{mg} / \mathrm{mL}$ YS and $200 \mu \mathrm{M}$ MJ enhanced the accumulation of MuA in MA root cultures. The treated roots were harvested in triplicate $24 \mathrm{~h}$ after treatment, as this showed the best response to single elicitor treatment. The samples were dried and analyzed for stilbene content [12].

\section{Sample extraction and preparation}

Root cultures were collected and dried in a hot air oven at $50{ }^{\circ} \mathrm{C}$ for two days and then powdered. Thirty milligrams of the dried powder was weighed and extracted using methanol as the solvent, assisted by sonication. The extract solution was collected after centrifugation at $6720 \times g$ for $10 \mathrm{~min}$. The plant residues were extracted three more times using the same procedure. The extract solutions were then combined and evaporated in a water bath $\left(50^{\circ} \mathrm{C}\right)$ to obtain the extract, which was then dissolved in $1 \mathrm{~mL}$ absolute methanol. All sample extracts were kept at $-20{ }^{\circ} \mathrm{C}$ until further analysis, both icELISA and HPLC analysis. All samples were analyzed in triplicate $(n=3)$.

\section{Stilbene determination and statistical analysis}

The concentration of MuA was analyzed by MuA-pAbbased icELISA described in a previous study [18]. The analytical procedure is described in detail in the electronic supplementary material (ESM). The HPLC method developed in our previous study [10] was applied to analyze the OXY and RES concentrations. The equipment and procedure are described in the ESM. All samples were analyzed in triplicate, and the results are presented as mean $\pm \mathrm{SD}$. The statistical significance of the differences between the control group and each treatment group were evaluated using a oneway analysis of variance (ANOVA), followed by the least significant difference (LSD) at the $p<0.01$ level.

\section{Results and discussion}

\section{MA root culture growth pattern and stilbene accumulation}

The evaluation of MA root culture growth in relation to stilbene production was performed for six weeks. As MA root growth is slow, the root samples were collected every week and the dried biomass was assessed. The root biomass gradually increased during the first 28 days of culture (the exponential growth phase), after which the growth reached the stationary phase (Table 1 ). The accumulation of MuA increased in tandem with the growth pattern. However, the MuA content decreased after the growth reached the stationary phase, whereas OXY and RES accumulation increased during this phase. The expression of UDPglycosyltransferase, which catalyzes the transfer of glucose molecules to RES, was high in the root cap meristem and correlated with cell division and root growth [19]. Therefore, 
Table 1 Time course for MuA content and DW of culture

\begin{tabular}{lllll}
\hline \multirow{2}{*}{$\begin{array}{l}\text { Time } \\
\text { (Weeks })\end{array}$} & Biomass & \multicolumn{2}{l}{ Stilbene accumulations $^{1}$} & \\
\cline { 3 - 5 } & $(\mathrm{g}$ DW/flask $)$ & MuA & OXY & RES \\
\hline 1 & $0.11 \pm 0.00^{\mathrm{a}}$ & $10.83 \pm 2.33^{\mathrm{a}}$ & $45.39 \pm 6.42^{\mathrm{a}}$ & $10.67 \pm 1.27^{\mathrm{a}, \mathrm{b}}$ \\
2 & $0.14 \pm 0.04^{\mathrm{a}, \mathrm{b}}$ & $14.38 \pm 0.75^{\mathrm{a}, \mathrm{b}}$ & $47.97 \pm 2.67^{\mathrm{a}}$ & $12.32 \pm 0.27^{\mathrm{a}, \mathrm{d}}$ \\
3 & $0.15 \pm 0.03^{\mathrm{a}, \mathrm{b}}$ & $18.47 \pm 2.11^{\mathrm{b}}$ & $43.14 \pm 3.60^{\mathrm{a}}$ & $10.12 \pm 0.23^{\mathrm{b}}$ \\
4 & $0.20 \pm 0.02^{\mathrm{b}}$ & $18.68 \pm 3.89^{\mathrm{b}}$ & $25.22 \pm 0.21^{\mathrm{b}}$ & $2.62 \pm 0.29^{\mathrm{c}}$ \\
5 & $0.20 \pm 0.04^{\mathrm{b}}$ & $12.79 \pm 2.90^{\mathrm{a}, \mathrm{b}}$ & $42.83 \pm 1.44^{\mathrm{a}}$ & $13.42 \pm 0.35^{\mathrm{d}}$ \\
6 & $0.22 \pm 0.03^{\mathrm{b}}$ & $15.02 \pm 1.56^{\mathrm{a}, \mathrm{b}}$ & $107.30 \pm 2.00^{\mathrm{c}}$ & $24.05 \pm 1.28^{\mathrm{e}}$ \\
\hline
\end{tabular}

${ }^{1}$ The contents of MuA are expressed in the unit of $\mathrm{mg} / \mathrm{g}$ DW, whereas OXY and RES are in the unit of $\mu \mathrm{g} / \mathrm{g}$ DW. The data were presented with the form of mean \pm SD. The same and different characters indicate the non-significant and significant difference, respectively, when data are compared between them in each column. Statistical significance is determined by one-way ANOVA, followed by Tukey HSD $(p<0.05)$
MuA production was high during the exponential growth phase, whereas glycosyltransferase expression decreased in the stationary growth phase, and the accumulation of nonglycosylated compounds (OXY and RES) increased. Thus, the roots were elicited for production of the stilbenes $\mathrm{MuA}$, OXY, and RES in the early stationary phase, i.e., the first 28 days, when the roots are usually ready for secondary metabolism.

\section{Effect of cell density on stilbene production by MA root culture}

To study the effect of cell density, the experiment was started by transferring $0.5,1,2,3$, and $6( \pm 0.1) \mathrm{g}$ fresh weight of root culture into $30 \mathrm{~mL}$ MS-NAA medium. After cultivation for four weeks, the roots were harvested to determine the stilbene accumulation by icELISA and HPLC. The root culture with an initial cell density of $1 \mathrm{~g} /$ flask reached the highest growth rate after four weeks (Table 2). When the root density increased further, the GI decreased. A higher initial inoculum/root density produced a lower GI because of the limited supply of oxygen and nutrients [20,21]. The stress condition of high root density and limited nutrients and oxygen increased the production and accumulation of stilbenes, with the highest contents of MuA, OXY, and RES observed in the root culture with an initial root density of 3 g/flask. Further increase in initial root density (6 g/flask) decreased the accumulated stilbene content. Hence, the $3 \mathrm{~g} /$ flask of initial inoculum density was considered the optimal condition for stilbene production.

\section{Effect of precursor feeding on stilbene accumulation in $M A$ root culture}

To investigate the effect of precursor feeding on stilbene production and accumulation, stilbenes were biosynthesized via phenylpropanoid metabolism [22, 23], using PHE and TYR as the precursors in the initial steps. Both amino acids and their combination were added to promote stilbene biosynthesis. After precursor feeding for 5, 10, 20, 40, and 50 days, the roots were harvested in triplicate, and the level of stilbene accumulation was investigated by icELISA and
Table 2 Effect of cell density on biomass and stilbenes accumulation

\begin{tabular}{|c|c|c|c|c|c|c|}
\hline \multicolumn{3}{|c|}{ Biomass $^{1}$} & \multirow[t]{2}{*}{$\mathrm{GI}^{2}$} & \multicolumn{3}{|c|}{ Stilbenes accumulations ${ }^{3}$} \\
\hline IFW & FFW & FDW & & $\mathrm{MuA}$ & OXY & RES \\
\hline 0.5 & $0.70 \pm 0.10^{\mathrm{a}}$ & $0.04 \pm 0.02^{\mathrm{a}}$ & $0.28 \pm 0.10^{\mathrm{a}}$ & $11.1 \pm 2.30^{\mathrm{a}}$ & $83.8 \pm 6.04^{\mathrm{a}}$ & $13.4 \pm 3.59^{\mathrm{a}}$ \\
\hline 1 & $2.60 \pm 0.28^{\mathrm{b}}$ & $0.2 \pm 0.13^{\mathrm{a}}$ & $0.61 \pm 0.04^{\mathrm{b}}$ & $10.2 \pm 0.48^{\mathrm{a}, \mathrm{c}}$ & $98.0 \pm 5.16^{\mathrm{a}}$ & $32.5 \pm 3.54^{\mathrm{b}}$ \\
\hline 2 & $4.46 \pm 0.28^{c}$ & $0.38 \pm 0.02^{\mathrm{b}}$ & $0.55 \pm 0.03^{\mathrm{b}}$ & $13.3 \pm 1.88^{\mathrm{a}}$ & $133 \pm 7.90^{b}$ & $38.4 \pm 1.51^{\mathrm{b}}$ \\
\hline 3 & $4.33 \pm 0.23^{\mathrm{c}}$ & $0.41 \pm 0.02^{\mathrm{b}}$ & $0.31 \pm 0.04^{\mathrm{a}}$ & $18.7 \pm 1.00^{\mathrm{b}}$ & $136 \pm 5.05^{\mathrm{b}}$ & $41.6 \pm 5.84^{\mathrm{b}}$ \\
\hline 6 & $8.66 \pm 0.58^{\mathrm{d}}$ & $0.79 \pm 0.01^{\mathrm{c}}$ & $0.31 \pm 0.05^{\mathrm{a}}$ & $6.65 \pm 0.62^{\mathrm{c}}$ & $88.6 \pm 10.5^{\mathrm{a}}$ & $14.0 \pm 0.63^{\mathrm{a}}$ \\
\hline
\end{tabular}

${ }^{1}$ The biomasses of MA roots were demonstrated with initial fresh weight (IFW), final fresh weight (FFW), and final DW (FDW), which all are in the unit of g/flask

${ }^{2}$ Growth index (GI) is calculated regarding the initial fresh weight (IFW) and final fresh weight (FFW)

${ }^{3}$ The contents of MuA are expressed in the unit of $\mathrm{mg} / \mathrm{g}$ DW, whereas OXY and RES are in the unit of $\mu \mathrm{g} / \mathrm{g}$ DW. The data were presented with the form of mean \pm SD. The same and different characters indicate the non-significant and significant difference, respectively, when data are compared between them in each column. Statistical significance is determined by one-way ANOVA, followed by Tukey HSD $(p<0.05)$ 
Fig. 1 Effects of precursor feeding [0.05 mM PHE, $0.03 \mathrm{mM}$ TYR, and their combination (PT)] on the accumulation of (a) MuA and (b) OXY and RES. The dried root for each treatment was subjected to stilbene extraction with methanol, the content of which was then determined using HPLC and icELISA. The experiment was performed in triplicate $(n=3)$, and the data are presented as the mean and SD indicated with an error bar. The significance of the mean difference between the precursor treatment and control (CON) was analyzed using a one-way ANOVA followed by the least significant difference at $p<0.01$, indicated by * a

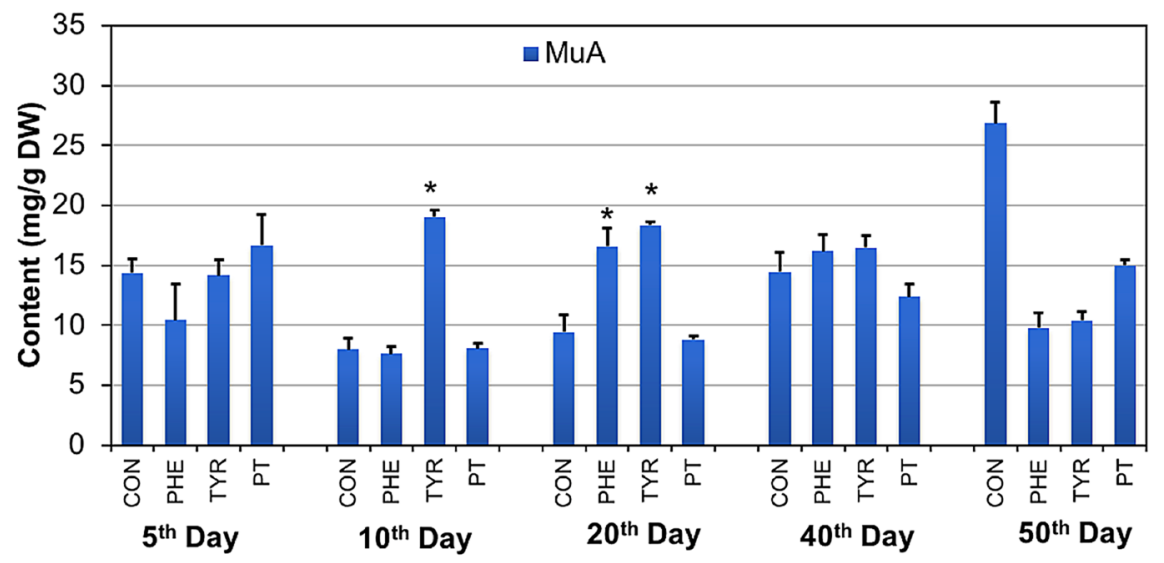

b

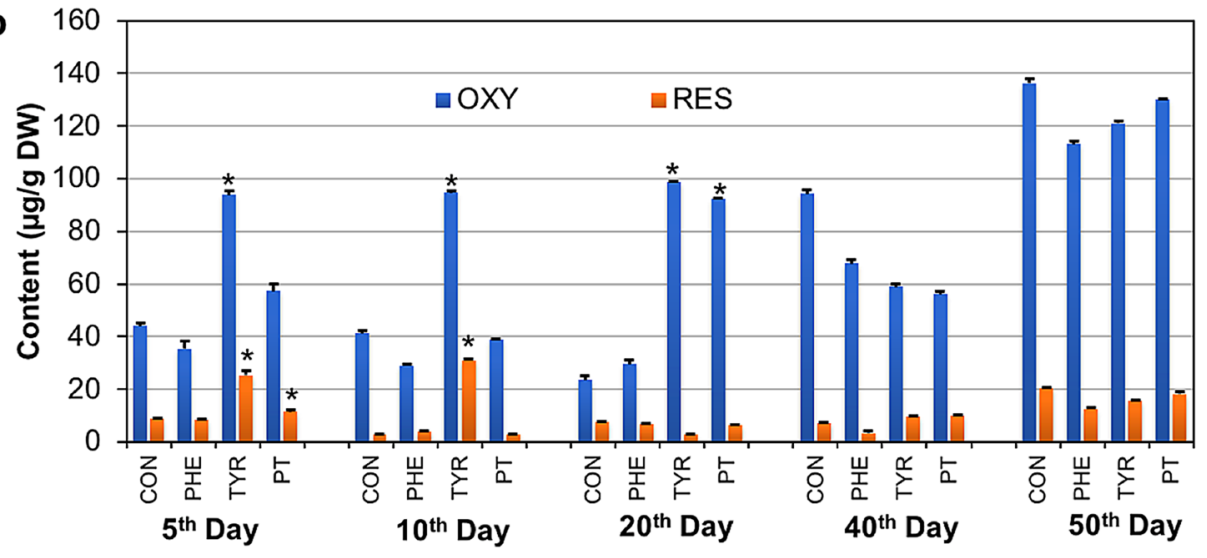

HPLC. Feeding $0.03 \mathrm{mM}$ TYR led to the highest accumulation of MuA (19.0 mg/g DW), OXY (94.7 $\mu \mathrm{g} / \mathrm{g} \mathrm{DW})$, and RES (31.1 $\mu \mathrm{g} / \mathrm{mg} \mathrm{DW})$ at day 10 after treatment (Fig. 1, ESM Table S1). The accumulation of these stilbenes decreased when the culture periods were longer than optimum. Although TYR and its combination with PHE can enhance the activity of phenylalanine ammonia-lyase (PAL) in vitro [24], the combination of PHE and TYR (PT) did not exert synergistic or additive effects on stilbene accumulation. TYR was transformed directly to p-coumaric acid by tyrosine ammonia-lyase (TAL), whereas PHE was enzymatically converted to p-coumaric acid by PAL and consequently to cinnamate 4-hydroxylase (4CH) [23]. p-Coumaric acid was further synthesized as a stilbene structure. PAL also displays TAL activity, converting TYR to p-coumaric acid [13]. Feeding TYR contributed more to stilbene synthesis in the early stage of growth. On the 50th day after precursor feeding and culture, the accumulation of all stilbenes was below that observed in the untreated MA root. Therefore, the advantage of precursor feeding lay in shortening the time to reach maximum stilbene production. However, TYR produced the highest accumulation of MuA, OXY, and RES after 40 days of immobilized MA cell culture [10]. This indicates that different types of culture may produce varied responses to precursor feeding.

\section{Effect of elicitors on stilbene accumulation in MA root culture}

According to a previous study, individual treatment of YS $(2 \mathrm{mg} / \mathrm{mL})$ and $\mathrm{MJ}(200 \mu \mathrm{M})$ significantly enhanced MuA production in MA root cultures after $24 \mathrm{~h}$ exposure, and the effects were higher than those observed in the roots treated with chitosan or salicylic acid [12]. Therefore, these two elicitors and the exposure period were selected to investigate the effect of co-elicitation on stilbene accumulation. MuA accumulation was significantly increased after treatment with YS $(2 \mathrm{mg} / \mathrm{mL})$ and its combination with MJ $(200 \mu \mathrm{M})$ for $24 \mathrm{~h}$, during which the roots produced $22.0 \pm 2.12$ and $30.3 \pm 2.68 \mathrm{mg} / \mathrm{g} \mathrm{DW}$ of $\mathrm{MuA}$, respectively. These MuA contents were 1.5- and 2.1-fold, respectively, higher than the MUA content in the control group $(14.7 \pm 1.27 \mathrm{mg} / \mathrm{g} \mathrm{DW})$. YS and its combination with MJ increased the OXY and RES contents in the treated roots (Fig. 2, ESM Table S2). The OXY accumulation in the roots treated with $2 \mathrm{mg} / \mathrm{mL}$ YS increased to $50.3 \pm 0.06 \mu \mathrm{g} / \mathrm{g} \mathrm{DW}$. When YS was combined with MJ $(200 \mu \mathrm{M})$, the OXY content further increased to $68.6 \pm 3.53 \mu \mathrm{g} / \mathrm{g} \mathrm{DW}$, compared with $26.5 \pm 1.79 \mu \mathrm{g} / \mathrm{g}$ DW in the control root. The abovementioned treatment also increased RES accumulation by 1.3- and 1.6-fold over that in the control group $(6.33 \pm 0.33 \mu \mathrm{g} / \mathrm{g} \mathrm{DW})$. Although this 
Fig. 2 Effects of $200 \mu \mathrm{M} \mathrm{MJ}$, $2 \mathrm{mg} / \mathrm{mL}$ YS, and their combinations [MY1 (50 $\mu \mathrm{M}$ MJ and $0.5 \mathrm{mg} / \mathrm{mL} \mathrm{YS}), \mathrm{MY} 2$ $(100 \mu \mathrm{M} \mathrm{MJ}$ and $1.0 \mathrm{mg} / \mathrm{mL}$ YS), and MY3 (200 $\mu \mathrm{M}$ MJ and $2 \mathrm{mg} / \mathrm{mL} \mathrm{YS}$ )] on (a) the accumulation of MuA and (b) OXY and RES. The dry root for each treatment was subjected to stilbene extraction using methanol, the content of which was then determined using HPLC and icELISA. The experiment was performed in triplicate $(n=3)$, and the data are presented as the mean and SD indicated with an error bar. The significance of the mean difference between the precursor treatment and control $(\mathrm{CON})$ was analyzed using a one-way ANOVA followed by the least significant difference at $p<0.01$, indicated by *

\section{a}

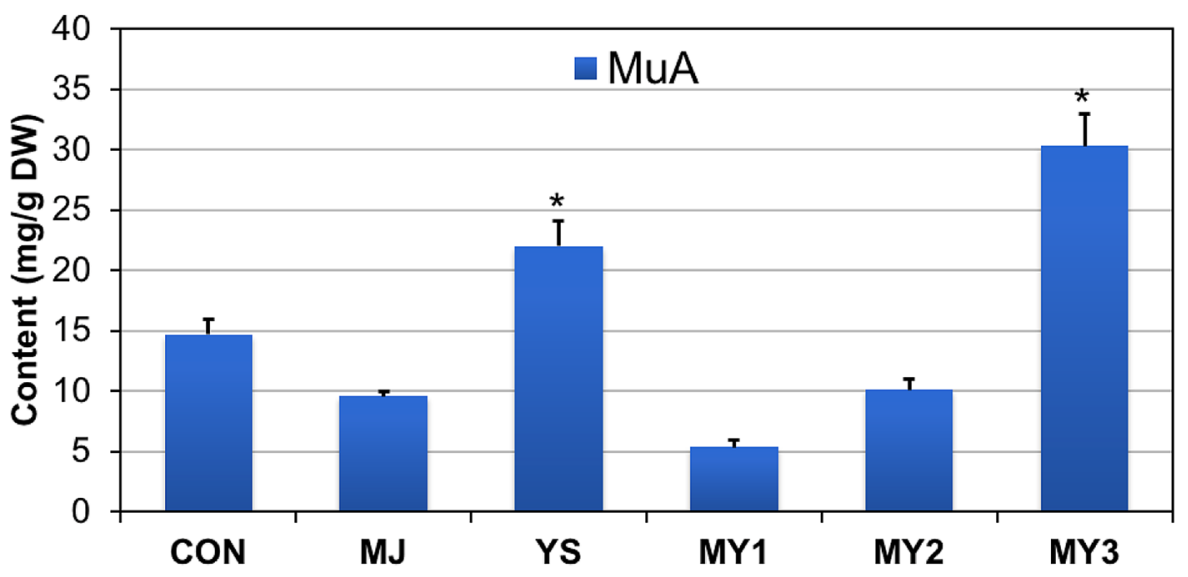

b

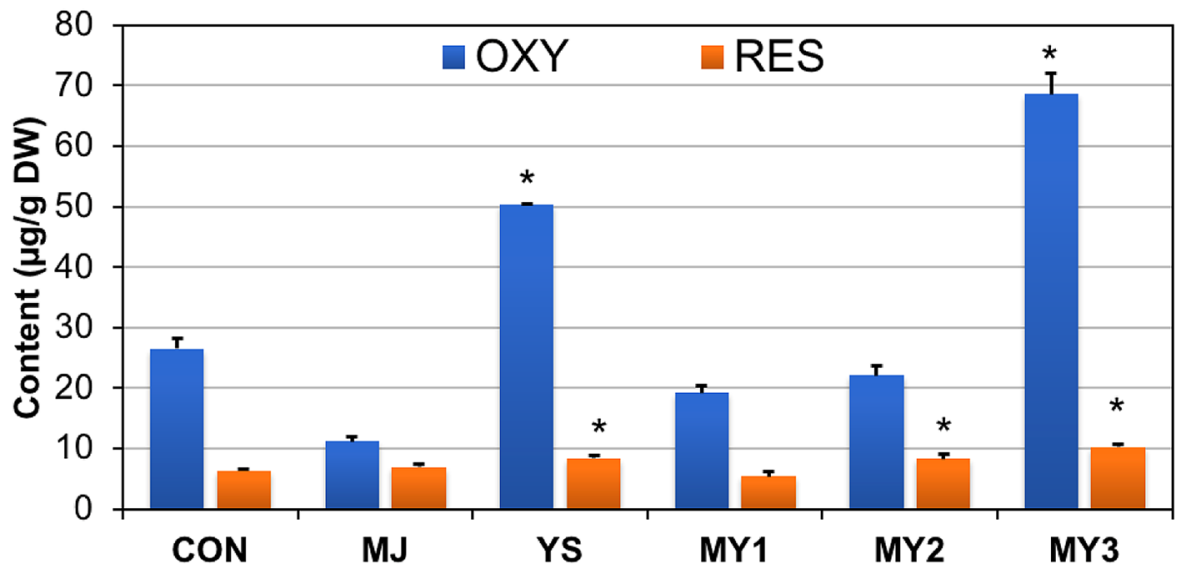

increase is statistically significant, the RES accumulation is still lower than that in the intact MA root [25] and Polygonum cuspidatum root [26]. Elicitors activate defense or stress-induced mechanisms that affect plant metabolism and usually enhance secondary metabolism. In a prior study, the treatment of grapevine cell cultures with $\mathrm{MJ}$ increased the expression of stilbene biosynthetic enzymes and the accumulation of trans-RES [27]. The combination of YS and $\mathrm{MJ}$ is usually useful in eliciting secondary plant metabolism via the phenylpropanoid pathway in Silybum marianum (L.) Gaertn cell cultures [28], Medicago truncatula cell suspension cultures [29], and MA cell suspension and root cultures [12]. A previous transcriptional analysis indicated that YS and MJ enhance different enzymes in the biosynthetic pathway. For example, YS induced a 45- and 14-fold increase in PAL and CHS transcripts, respectively, in cell suspension cultures of the model legume M. truncatula [29]. In addition, MJ increased the transcription of stilbene synthase (STS) in grapevine cell cultures [27]. The co-treatment of MJ and YS significantly enhanced the accumulation of MuA, OXY, and RES in an immobilized MA cell culture [10]. This raises the possibility that MJ and YS induce different enzymes involved in stilbene biosynthesis, and that their combination provides co-elicitation. Therefore, the co-elicited MA root culture produced a comparable amount of $\mathrm{MuA}$ to the intact root, which accumulated MuA at 5.24-24.32 mg/g DW [18] and $0.093-47.196 \mathrm{mg} / \mathrm{g}$ [30]. The stilbene productivity of an MA cell suspension culture was also enhanced by treatment with YS, MJ, chitosan, and salicylic acid, which increased $\mathrm{MuA}$ accumulation to $6.9-33.0 \mathrm{mg} / \mathrm{g}$ DW compared with $9.9 \mathrm{mg} / \mathrm{g} \mathrm{DW}$ in the control culture [12]. The stilbene production of the MA root culture in response to YS and MJ was less than that of the cell suspension culture, which may be related to the different cell structures. The co-treatment of cell immobilization and elicitation (MJ and YS) of MA cells increased both intracellular and secreted stilbenes, yielding a total content of $70.48 \mathrm{mg} / \mathrm{g}$ DW [10]. In addition, other substances (cyclodextrin, salicylic acid, and ethylene) have also been shown to stimulate RES production in the Vitis vinifera cultivar Monastrell [31], but YS and MJ exhibited more efficient elicitation than chitosan and salicylic acid in the MA root culture [12].

Overall, elicitation makes MA root a sustainable and environmentally friendly source of stilbenes for pharmaceutical and cosmeceutical production. The co-elicitation treatment effectively enhanced the accumulation of MuA, 
RES, and OXY. Further investigation using an integrated approach is required to elucidate the interactions between inoculum density, precursor feeding, and elicitors to achieve maximum productivity of these stilbenes. Although the accumulation of RES and OXY was much improved, their contents were much lower than that of MuA. However, MuA can be bio-converted to OXY by beta-glucosidase [32]. Thus, MuA can be a source of OXY that exhibits various pharmacological activities, including anti-COVID-19 properties.

Acknowledgements This research was supported by the Graduate School, Khon Kaen University, Faculty of Pharmaceutical Sciences, Khon Kaen University, the National Research Council of Thailand (NRCT), and the Thailand Science Research and Innovation (Grant no. IRN61W0005).

\section{Compliance with ethical standards}

Conflict of interest The authors declare that they have no competing interests.

Human and animal rights statement This article does not contain any studies with human participants or animals performed by any of the authors.

\section{References}

1. Chen YC, Tien YJ, Chen CH, Beltran FN, Amor EC, Wang RJ, Wu DJ, Mettling C, Lin YL, Yang WC (2013) Morus alba and active compound oxyresveratrol exert anti-inflammatory activity via inhibition of leukocyte migration involving MEK/ERK signaling. BMC Complement Altern Med 13:45

2. Kim JK, Park KT, Lee HS, Kim M, Lim YH (2012) Evaluation of the inhibition of mushroom tyrosinase and cellular tyrosinase activities of oxyresveratrol: comparison with mulberroside A. J Enzyme Inhib Med Chem 27:495-503

3. Lorenz P, Roychowdhury S, Engelmann M, Wolf G, Horn TF (2003) Oxyresveratrol and resveratrol are potent antioxidants and free radical scavengers: effect on nitrosative and oxidative stress derived from microglial cells. Nitric Oxide 9:64-76

4. Choi HY, Lee JH, Jegal KH, Cho IJ, Kim YW, Kim SC (2016) Oxyresveratrol abrogates oxidative stress by activating ERK-Nrf2 pathway in the liver. Chem Biol Interact 245:110-121

5. Wei H, Zhu JJ, Liu XQ, Feng WH, Wang ZM, Yan LH (2016) Review of bioactive compounds from root barks of Morus plants (Sang-Bai-Pi) and their pharmacological effects. Cogent Chem 2:1212320

6. Wahedi HM, Ahmad S (2020) Abbasi SW (2020) Stilbene-based natural compounds as promising drug candidates against COVID19. J Biomol Struct Dyn 10(1080/07391102): 1762743

7. Li YQ, Li ZL, Zhao WJ, Wen RX, Meng QW, Zeng Y (2006) Synthesis of stilbene derivatives with inhibition of SARS coronavirus replication. Eur J Med Chem 41:1084-1089

8. Chuanasa T, Phromjai J, Lipipun V, Likhitwitayawuid K, Suzuki M, Pramyothin P, Hattori M, Shiraki K (2008) Anti-herpes simplex virus (HSV-1) activity of oxyresveratrol derived from Thai medicinal plant: mechanism of action and therapeutic efficacy on cutaneous HSV-1 infection in mice. Antiviral Res 80:62-70
9. Filardo S, Di Pietro M, Mastromarino P, Sessa R (2020) Therapeutic potential of resveratrol against emerging respiratory viral infections. Pharmacol Ther 214:107613

10. Inyai C, Boonsnongcheep P, Komaikul J, Sritularak B, Tanaka H, Putalun W (2019) Alginate immobilization of Morus alba L. cell suspension cultures improved the accumulation and secretion of stilbenoids. Bioprocess Biosyst Eng 42:131-141

11. Komaikul J, Kitisripanya T, Likhitwitayawuid K, Sritularak B, Tanaka H, Putalun W (2019) Improvement of stilbenoid production by 2-hydroxypropyl-beta-cyclodextrin in white mulberry (Morus alba L.) callus cultures. Nat Prod Res 33:2762-2769

12. Komaikul J, Kitisripanya T, Tanaka H, Sritularak B, Putalun W (2015) Enhanced mulberroside a production from cell suspension and root cultures of Morus alba using elicitation. Nat Prod Commun 10:1253-1256

13. Ramirez-Estrada K, Vidal-Limon H, Hidalgo D, Moyano E, Golenioswki M, Cusido RM, Palazon J (2016) Elicitation, an effective strategy for the biotechnological production of bioactive high-added value compounds in plant cell factories. Molecules $21: 182$

14. Halder M, Sarkar S, Jha S (2019) Elicitation: A biotechnological tool for enhanced production of secondary metabolites in hairy root cultures. Eng Life Sci 19:880-895

15. Ouyang J, Wang XD, Zhao B, Wang YC (2005) Enhanced production of phenylethanoid glycosides by precursor feeding to cell culture of Cistanche deserticola. Process Biochem 40:3480-3484

16. Murthy HN, Lee EJ, Paek KY (2014) Production of secondary metabolites from cell and organ cultures: strategies and approaches for biomass improvement and metabolite accumulation. Plant Cell Tiss Org 118:1-16

17. Yu O, Jez JM (2008) Nature's assembly line: biosynthesis of simple phenylpropanoids and polyketides. Plant J 54:750-762

18. Komaikul J, Kitisripanya T, Tanaka H, Sritularak B, Putalun W (2014) Development of an enzyme-linked immunosorbent assay for specific detection of mulberroside A in mulberry (Morus alba L.) using anti-mulberroside A polyclonal antibody. Food Anal Method 7:58-63

19. Woo HH, Orbach MJ, Hirsch AM, Hawes MC (1999) Meristemlocalized inducible expression of a UDP-glycosyltransferase gene is essential for growth and development in pea and alfalfa. Plant Cell 11:2303-2315

20. Min JY, Jung HY, Kang SM, Kim YD, Kang YM, Park DJ, Prasad DT, Choi MS (2007) Production of tropane alkaloids by smallscale bubble column bioreactor cultures of Scopolia parviflora adventitious roots. Bioresour Technol 98:1748-1753

21. Reis RV, Borges APP, Chierrito TPC, de Souto ER, de Souza LM, Iacomini M, de Oliveira AJB, Goncalves RAC (2011) Establishment of adventitious root culture of Stevia rebaudiana Bertoni in a roller bottle system. Plant Cell Tiss Org 106:329-335

22. Emiliani G, Fondi M, Fani R, Gribaldo S (2009) A horizontal gene transfer at the origin of phenylpropanoid metabolism: a key adaptation of plants to land. Biol Direct 4:7

23. Dubrovina AS, Kiselev KV (2017) Regulation of stilbene biosynthesis in plants. Planta 246:597-623

24. Cui JD, Jia SR, Sun AY (2008) Influence of amino acids, organic solvents and surfactants for phenylalanine ammonia lyase activity in recombinant Escherichia coli. Lett Appl Microbiol 46:631-635

25. Zhou J, Li SX, Wang W, Guo XY, Lu XY, Yan XP, Huang D, Wei BY, Cao L (2013) Variations in the levels of mulberroside A, oxyresveratrol, and resveratrol in mulberries in different seasons and during growth. Sci World J 2013:380692

26. Vastano BC, Chen Y, Zhu N, Ho CT, Zhou Z, Rosen RT (2000) Isolation and identification of stilbenes in two varieties of Polygonum cuspidatum. J Agric Food Chem 48:253-256

27. Belhadj A, Telef N, Saigne C, Cluzet S, Barrieu F, Hamdi S, Merillon JM (2008) Effect of methyl jasmonate in combination 
with carbohydrates on gene expression of PR proteins, stilbene and anthocyanin accumulation in grapevine cell cultures. Plant Physiol Biochem 46:493-499

28. Sanchez-Sampedro MA, Fernandez-Tarrago J, Corchete P (2005) Yeast extract and methyl jasmonate-induced silymarin production in cell cultures of Silybum marianum (L.) Gaertn. J Biotechnol 119:60-69

29. Suzuki H, Reddy MS, Naoumkina M, Aziz N, May GD, Huhman DV, Sumner LW, Blount JW, Mendes P, Dixon RA (2005) Methyl jasmonate and yeast elicitor induce differential transcriptional and metabolic re-programming in cell suspension cultures of the model legume Medicago truncatula. Planta 220:696-707

30. Piao SJ, Chen LX, Kang N, Qiu F (2011) Simultaneous determination of five characteristic stilbene glycosides in root bark of Morus albus L. (Cortex mori) using high-performance liquid chromatography. Phytochem Anal 22:230-235
31. Belchi-Navarro S, Almagro L, Sabater-Jara AB, Fernandez-Perez F, Bru R, Pedreno MA (2013) Induction of trans-resveratrol and extracellular pathogenesis-related proteins in elicited suspension cultured cells of Vitis vinifera cv Monastrell. J Plant Physiol 170:258-264

32. Ko JA, Park JY, Kwon HJ, Ryu YB, Jeong HJ, Park SJ, Kim CY, Oh HM, Park CS, Lim YH, Kim D, Rho MC, Lee WS, Kim YM (2014) Purification and functional characterization of the first stilbene glucoside-specific beta-glucosidase isolated from $\mathrm{Lac}$ tobacillus kimchi. Enzyme Microb Technol 67:59-66

Publisher's Note Springer Nature remains neutral with regard to jurisdictional claims in published maps and institutional affiliations. 